\title{
LEFT VENTRICULAR FUNCTION CHANGES IN SEVERE ANEMIA BY ECHOCARDIOGRAPHY: A CORRELATION STUDY
}

Farquana Qushnood ${ }^{1}$, Sanjay B. Bhagoji², Shireen Swaliha Quadri ${ }^{3}$, Zaheera Sultana $S^{4}$, Salim A. Dhundasi ${ }^{5}$, K. K. Das ${ }^{6}$

\section{HOW TO CITE THIS ARTICLE:}

Farquana Qushnood, Sanjay B. Bhagoji, Shireen Swaliha Quadri, Zaheera Sultana S, Salim A. Dhundasi, K. K. Das. "Left Ventricular Function Changes in Severe Anemia by Echocardiography: A Correlation Study". Journal of Evolution of Medical and Dental Sciences 2014; Vol. 3, Issue 11, March 17; Page: 2879-2885,

DOI: $10.14260 /$ jemds/2014/2219

ABSTRACT: BACKGROUND AND OBJECTIVES: In the last decade, anemia is recognized as an important cause of morbidity, a factor limiting physical activity, responsible for a poor quality of life, and a predictor of unfavorable outcomes. Anemia is emerging as a potential contributor to the development and progression of cardiovascular disease. Hence the present study was undertaken to correlate echocardiographic changes with anemic status. METHODOLOGY: Present study was conducted in Al-Ameen Medical College and Government district Hospital, Bijapur. 31 anemic patients (aged 18-40 yrs.) with $\mathrm{Hb} \leq 7 \mathrm{gm}$. \% were selected. All anemic patients were subjected for hemoglobin estimation and M mode 2D Echocardiography. Echocardiographic parameters- IVSTd, LVIDd, LVPWd, IVSTs, LVIDs, LVPWS, EDV, ESV, SV, SI, CI, EF, FS were studied and correlated with hemoglobin levels. Statistical analysis was done by correlation analysis. RESULTS: LVPWd, IVSTs, IVStd, LVPWs did not correlate with hemoglobin levels. LVIDd, LVIDs, EDV, ESV, SV, SI, CI were negatively correlated with hemoglobin levels in anemic patients. No correlation observed for EF, FS. INTERPRETATION AND CONCLUSION: The findings of negative correlation of echo parameters with anemic status may be as a consequence of hyperdynamic circulatory state leading to vascular and cardiac changes. These changes are mainly due to increased preload, decreased afterload, changes in cardiac geometry. These factors increase systolic wall stress and over time lead to LV systolic dysfunction.

KEYWORDS: Chronic anemia, left ventricle, hemoglobin, echocardiography.

INTRODUCTION: In the last decade, anemia is recognized as an important cause of morbidity, a factor limiting physical activity, responsible for a poor quality of life, and a predictor of unfavourable outcomes. Anemia is emerging as a potential contributor to the development and progression of cardiovascular disease. Extreme alterations in blood count as in anemia are known to cause circulatory changes and if these alterations persist, there occurs adaptations of cardiac geometry. ${ }^{1}$

Anemia significantly alters circulatory dynamics and so burdens circulatory system.

Echocardiography is a valuable non-invasive diagnostic test for the assessment of cardiovascular system. Only few studies have assessed LV function by echocardiography. Studies conducted to assess LV function by non-invasive techniques show LV dysfunction in chronic severe anemia.

The LV internal dimensions in systole and diastole, end systolic and end diastolic volume index, and other performance indices are deranged in all anemic patients. Clinical studies suggest that correction of anemia causes regression of left ventricular hypertrophy, may improve exercise capacity and decrease adverse outcomes in patients with congestive heart failure. ${ }^{2}$ Hence the present study was undertaken to correlate echocardiographic changes with anemic status. 
MATERIALS AND METHODS: The present study was conducted in the department of physiology AlAmeen Medical College, Bijapur. Thirty one (31) patients (20 females, 11 males) with severe anemia ( $\mathrm{Hb} \leq 7 \mathrm{gm} \%$ ), between 18yrs to 40 yrs. age visiting Al-Ameen medical college hospital, Bijapur and District Hospital Bijapur were selected.

Patients presenting with acute blood loss, pregnancy, hypertension, any underlying heart disease, COPD, patients with hyperdynamic circulatory states like hyperthyroidism, beriberi, AV fistulas were excluded from the study.

All anemic patients underwent history taking and a thorough clinical examination. Their hemoglobin levels were estimated and were subjected for $M$ mode two dimensional Echocardiography. Transthoracic Echocardiography was done using PHILIPS Envisor C (model no MCMD02AA) diagnostic Ultrasound System using 3.5 MHz transducer, probe (PA 4- 2) by physician experienced in Echocardiography. Measurements of the LV cavity dimension and wall thickness are derived from M-mode recordings and are made according to the recommendations of the American Society of Echocardiography (ASE) at end diastole and end systole. ${ }^{3}$

Echocardiographic parameters studied were: Interventricular septum thickness at end diastole and end systole (IVSTd \& IVSTs respectively), Left ventricular inner dimension at end diastole and end systole (LVIDd \& LVIDs respectively), Posterior wall thickness at end diastole and end systole (LVPWd \& LVPWs respectively). 2D echocardiography is significantly superior to M-mode approaches for the measurement of cardiac chamber volumes and EF. End diastolic volume (EDV), End systolic volume (ESV), Stroke Volume (SV) were obtained. Derived parameters of Systolic function includes: Left Ventricular Ejection Fraction (LVEF), Fractional Shortening (FS). Cardiac output (CO) was calculated as CO = HR x SV. Stroke index (SI) and Cardiac index (CI) were calculated.

Correlation analysis (using Graph pad Prism 5 statistical software) was applied to correlate hemoglobin levels and echocardiographic parameters. A 'p' value $<0.05$ was considered statistically significant.

\section{RESULTS:}

\begin{tabular}{|l|c|}
\hline \multicolumn{1}{|c|}{ PARAMETER } & $\begin{array}{c}\text { ANEMIC PATIENTS (n = 31) } \\
\text { MEAN } \pm \text { SEM }\end{array}$ \\
\hline AGE (yrs.) & $31.8 \pm 1.33$ \\
\hline $\mathrm{Hb}$ (gm./dl) & $4.958 \pm 0.22$ \\
\hline $\mathrm{PR}($ beats $/ \mathrm{min})$ & $88.06 \pm 1.08$ \\
\hline SBP $(\mathrm{mmHg})$ & $109.29 \pm 1.39$ \\
\hline $\mathrm{DBP}(\mathrm{mmHg})$ & $66.58 \pm 1.38$ \\
\hline
\end{tabular}

TABLE NO. 1: Shows the mean \pm SEM of Age, Hb, $\mathrm{PR}, \mathrm{SBP}, \mathrm{DBP}$ in anemic patients

\begin{tabular}{|c|l|c|c|c|}
\hline \multirow{2}{*}{ Sl. No. } & \multirow{2}{*}{ PARAMETER } & \multicolumn{3}{|c|}{ ANEMIC PATIENTS (N=31) } \\
\cline { 3 - 5 } & & $\mathbf{r}$ & $\mathbf{p}$ & $\mathbf{S} / \mathbf{N S}$ \\
\hline 1 & Hb vs. IVSTd & -0.341 & 0.06 & NS \\
\hline 2 & Hb vs. LVIDd & -0.544 & 0.001 & S $^{* *}$ \\
\hline 3 & Hb vs. LVPWd & -0.287 & 0.11 & NS \\
\hline
\end{tabular}




\begin{tabular}{|c|l|c|c|c|}
\hline 4 & Hb vs. IVSTs & -0.256 & 0.16 & NS \\
\hline 5 & Hb vs. LVIDs & -0.364 & 0.04 & $\mathrm{~S}^{*}$ \\
\hline 6 & Hb vs. LVPWs & -0.128 & 0.49 & NS \\
\hline 7 & Hb vs. EDV & -0.525 & 0.002 & $\mathrm{~S}^{* *}$ \\
\hline 8 & Hb vs. ESV & -0.415 & 0.02 & $\mathrm{~S}^{*}$ \\
\hline 9 & Hb vs. SV & -0.571 & $<0.001$ & $\mathrm{~S}^{* * *}$ \\
\hline 10 & Hb vs. SI & -0.669 & $<0.0001$ & $\mathrm{~S}^{* * *}$ \\
\hline 11 & Hb vs. CI & -0.641 & 0.0001 & $\mathrm{~S}^{* * *}$ \\
\hline 12 & Hb vs. EF & 0.062 & 0.73 & NS \\
\hline 13 & Hb vs. FS & 0.07 & 0.70 & NS \\
\hline
\end{tabular}

Thirty one patients with severe anemia having hemoglobin concentration less than $7 \mathrm{gm} . / \mathrm{dl}$, between 18 and 40 yrs. of age were selected for this study. Correlation analysis was done to correlate various parameters among themselves.

Table No. 1 shows the mean \pm SEM of Age, Hb, PR, SBP, DBP in anemic patients.

The mean Hb level was 4.958 gm.\%. Correlation coefficient ( $r$ ) and 'p' values of correlation analysis of anemic patients were calculated. Table No. 2 shows the correlation analysis of Hb with various Echo parameters in anemic patients.

The correlation between IVSTd and hemoglobin was not statistically significant; $(r=-0.341$, $\mathrm{p}>0.05)$. The LVIDd showed statistically significant negative correlation with hemoglobin levels $(\mathrm{r}=-0.544, \mathrm{p}<0.01)$. There was no significant correlation between LVPWd and hemoglobin $(\mathrm{r}=-0.287$, $\mathrm{p}>0.05)$. Similar results were obtained for IVSTs and hemoglobin $(r=-0.256, p>0.05)$. The LVIDs showed statistically significant negative correlation with hemoglobin $(r=-0.364, p<0.05)$. LVPWs and hemoglobin were not correlated with a statistical significance $(r=-0.128, p>0.05)$.

The End diastolic volume (EDV) and hemoglobin levels were negatively correlated which was statistically significant $(\mathrm{r}=-0.525, \mathrm{p}<0.01)$. Significant negative correlation was found between ESV and hemoglobin levels $(\mathrm{r}=-0.415, \mathrm{p}<0.05)$. A significant negative correlation found between stroke volume and hemoglobin levels; $(\mathrm{r}=-0.571, \mathrm{p}<0.001)$. SI and CI were negatively correlated with $\mathrm{Hb}$ levels with statistical significance $(r=-0.669, \mathrm{p}<0.0001) \&(\mathrm{r}=-0.641, \mathrm{p}<0.001)$ respectively.

There was no statistically significant correlation between EF and hemoglobin ( $r=0.062$, $p>0.05)$. Similar results were found for FS and hemoglobin ( $r=0.07, p>0.05)$.

DISCUSSION: It is suggested that cardiac evidence of chronic anemia generally appears when the circulating hemoglobin level is less than $7 \mathrm{gm} / \mathrm{dl} .{ }^{40}$ The transition from a high-output (compensated) cardiac state to a state of LV dysfunction (decompensated) appears to begin at a hemoglobin level of approximately $7 \mathrm{~g} / \mathrm{dl}$. As the hemoglobin level drops further, so does the LV function. ${ }^{4}$

The hematocrit levels were inversely related to end diastolic diameters in study conducted by Stritzke J et al. in 2007.1 28 patients with iron deficiency anemia were investigated in respect to their parameters of left ventricular (LV) systolic and diastolic function. Changes in hemodynamic parameters were observed before and after treatment in 14 subjects with moderate to severe anemia and then were compared with those of normal subjects. 
It was found that the LV diastolic diameter decreased after treatment in anemic patients (Takahashi M et al.1990).5 LVIDd correlated negatively in our study. It may be due to as a consequence of chronic anemia. The finding of our study is in agreement with other studies. In chronic severe anemia the LV preload is significantly higher and after load is lower due to reduced systemic vascular resistance. The decreased after load may be due to peripheral vasodilatation related to anemia. ${ }^{6}$ With decreased afterload, the venous return (preload) and left ventricular (LV) filling increase, leading to increased LV end-diastolic fiber length. An increased left ventricular end diastolic dimension can be attributed towards the role of Frank Starling mechanism in the hyper dynamic state of chronic anemia. ${ }^{7}$ In chronic anemia the hemodynamic adaptations causes changes in cardiac geometry.

Significant reduction in LVIDs dimensions were observed in anemic patients after therapy $(2.66 \pm 0.45 \mathrm{cms})$ as compared to pre therapy $(2.81 \pm 0.53 \mathrm{cms})$ in a study on 30 patients with iron deficiency anemia to evaluate myocardial function by $2 \mathrm{D}$ echocardiography before and after total dose iron therapy. (JF Alvares et al. 2000). ${ }^{8}$ Hence the values improved with increase in hemoglobin levels. Our finding of increased LVIDs is in accordance with other studies mentioned above. It may be due to the result of increased volume overload which in the long term causes hemodynamic alterations leading to gradual development of cardiac enlargement and LV hypertrophy. This LVH is eccentric, characterized by increased LV internal dimensions. ${ }^{9}$

A study conducted on 30 patients (aged 3yrs to 14 yrs.) with IDA estimated the left ventricular contractility by echocardiographic indexes before and after iron therapy. It was observed that EDV index in anemic patients was significantly lower as compared to controls and after therapy the values were similar to that of controls implying rise in Hb levels lowered EDV (Riku $\mathrm{H}$ et al 1999). ${ }^{6}$

Our observation of a negative correlation between EDV and hemoglobin levels in anemic patients indicates rise in EDV with fall in $\mathrm{Hb}$ levels, which in turn may be attributed towards hyperdynamic state in chronic anemia which causes an increased LV preload. As the preload increases the left ventricular filling increases leading to increased LV end-diastolic volume in order to maintain a high stroke work. ${ }^{9}$ The rise in ESV with fall in Hb levels in found in our study may be due to early feature of onset of impaired left ventricular function in these patients. Chronic anemia is known to cause systolic dysfunction especially when the hemoglobin concentration falls below 6 gm./dl.

Higher stroke index was found in anemic patients when compared with healthy controls (RB Panwar et al. 1991). ${ }^{10}$ An increase in SV was accompanied by an increase in LV preload in a study where in changes in hemodynamic parameters were observed before and after treatment in 14 anemic subjects (Takahashi M et al 1990). ${ }^{5}$ In a study on 687 women and 648 men, aged 25-74 years, participating in the third population-based MONICA Augsburg study, it was observed that the stroke volume correlated inversely with haematocrit levels (Stritzke J et al.2007). ${ }^{1}$

The findings of our study are in accordance with other studies. The negative correlation of SV and SI with HB levels in anemic patients may be attributed towards hyperdynamic state in chronic anemia which causes an increased LV preload and lower after load. The decreased after load may be due to peripheral vasodilatation related to anemia. ${ }^{6}$

As SV and SI is associated with increase in LVIDd in our study, once again t may be due to the Frank Starling mechanism that operates in the hyper dynamic state of chronic anemia. ${ }^{9}$ Stroke 
volume can also be increased in chronic anemia even when heart rate is rapid. ${ }^{11}$ An increase in SV is an important determinant of high cardiac output in chronic anemia, because it parallels the severity of anemia. ${ }^{5}$

The EF and FS which are considered to be an indices of systolic function being lower in anemic patients may indicate that the systolic function in these patients is slightly compromised.

However they did not correlate with $\mathrm{Hb}$ levels in our study, which may be due to well compensated state of anemic patients.

Significantly $(\mathrm{p}<0.001)$ higher cardiac index was found in anemic patients $(7.3 \pm 3.0$ $\mathrm{L} / \mathrm{min} / \mathrm{m}^{2}$ ) when compared with that of normal subjects $\left(3.8 \pm 1.6 \mathrm{~L} / \mathrm{min} / \mathrm{m}^{2}\right)$ (Bahl VK et al. 1992). ${ }^{7}$ A study was conducted by Schafer GE et al in 2002. Patients were divided into two groups: in patients with severe anemia ( $\mathrm{Hb} 5.7 \pm 0.6 \mathrm{~g} / \mathrm{dl} ; \mathrm{n}=7)$. Cardiac index was higher $\left(4.8 \pm 0.4 \mathrm{~L} / \mathrm{min} / \mathrm{m}^{2}<0.01\right)$ compared with the other group $\left(\mathrm{Hb} 9.8+/-1.7 \mathrm{~g} / \mathrm{dl} ; \mathrm{n}=24 ; \mathrm{CI} 3.9 \pm 1.1 \mathrm{~L} / \mathrm{min} / \mathrm{m}^{2}\right)$. It was found that the increase of cardiac index caused by anemia correlated with increased stroke volume and heart rate and lowered pulmonary and peripheral resistance. Patients with severe anemia showed a tendency to an impaired cardiac index below $\mathrm{Hb}<5-6 \mathrm{gm} . / \mathrm{dl} .{ }^{12}$ It was also found that the cardiac index decreased in patients with IDA after therapy when compared with before therapy. ${ }^{6}$

The increased CI in anemic patients is probably due to reduced oxygen supply to the tissues found in chronic anemia that causes hemodynamic mechanisms to operate to compensate for anemia. Increased cardiac output is the main hemodynamic factor mediated by lower afterload, increased preload and positive chronotropic effects. The increased volume load is a reflection of the FrankStarling mechanism and an increase in heart rate. ${ }^{13}$ The negative correlation of CI with hemoglobin levels in anemic patients may be due to as a consequence of chronic anemia. This increased CI has mainly contributed to the high output state of chronic anemia.

Over all the cardiac changes are mainly due to increased preload, decreased afterload, positive chronotropic effects and changes in cardiac geometry. These changes may be attributed to ventricular remodelling that occurs following sustained hemodynamic overloading of the heart which may be due to volume overload. ${ }^{14}$ Chronic anemia causes a long lasting volume overload which results in ventricular dilatation. These factors increases systolic wall stress and result in eccentric left ventricular hypertrophy characterized by increased LV dimensions. ${ }^{14}$ Over time the stress of volume overload weakens the left ventricle and leads to LV systolic dysfunction. These hemodynamic changes vary in severity with the degree of anemia. These changes in chronic severe anemia if continued for prolonged period may finally lead to cardiac failure. 15

CONCLUSION: Our findings of significant negative correlation of LVIDd, LVIDs, EDV, ESV, SV, SI, CI, with hemoglobin levels in anemic patients, may be as consequence of hyperdynamic circulatory state leading to vascular and cardiac changes. The cardiac changes are mainly due to increased preload, decreased afterload, positive chronotropic effects and changes in cardiac geometry. The increased preload conditions found in anemic patients in our study may be attributed to the Frank Starling mechanism that is operating in hyperdynamic state of chronic anemia. Over time the stress of volume overload weakens the left ventricle and leads to LV systolic dysfunction. These hemodynamic changes may vary in severity with the degree of anemia. 


\section{REFERENCES:}

1. Stritzke J, Mayer B, Lieb W, Luchner A, Döring A, Hense HW, Schunkert H. Hematocrit levels and left ventricular geometry: Results of the MONICA Augsburg Echocardiographic Substudy. J Hpertens 2007 Jun; 25 (6):1301-9.

2. NAAC- National anemia action council (Online) 2008 May. [As accessed on $8^{\text {th }}$ Jun 2011]. Available from URL: http:// www.anemia.Org/professional/index .php.

3. Ronal G.Grainger, David Allison, Andreas Adam, Adrian K Dixon. Grainger and Allison's

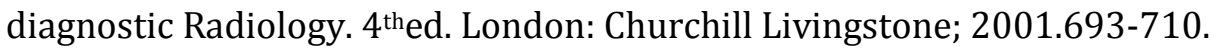

4. Nikita Hegde, Michael W. Rich, Charina Gayomali. The Cardiomyopathy of Iron Deficiency. Tex Heart Inst J 2006; 33: 340-4.

5. Takahashi M, Kurokawa S, Tsuyusaki T, Kikawada R. Studies of hyperkinetic circulatory state in chronic anemia. J Cardiol 1990; 20 (2):331-9.

6. Hayashi R, Ogawa S, Watanabe Z, Yamamoto M. Cardiovascular function before and after iron therapy by echocardiography in patients with iron deficiency anemia. Pediatr Int 1999; 41: 13-7.

7. Bahl VK, Malhotra OP, Kumar D, Agarwal R, Goswami KC, Bajaj R, et al. Non-invasive assessment of systolic and diastolic left ventricular function in patients with chronic severe anemia: A combined M-mode, two-dimensional, and Doppler echocardiographic study. Am Heart J 1992; 124: 1516-23.

8. Alvares JF, Oak JL, Pathare AV. Evaluation of cardiac function in iron deficiency anemia before and after total dose iron therapy. J Assoc Physicians India 2000; 48: 204-6.

9. Metivier F, Marchais SJ, Guerin AP, Pannier B, London GM. Pathophysiology of anaemia: Focus on the heart and blood vessels. Nephrol Dial Transplant 2000; 15 Suppl 3: 14-8.

10. RB Panwar, R Goyal, RP Shukla, SN Misra. Assessment of left ventricular function in chronic severe anemia. An echocardiographic study. JAPI 1991; 39(9): 673-674.

11. Heriburt Schunkert, Hans W. Hense. A Heart price to pay for anaemia. Nephrol Dial Transplant 2001; 16: 445-448.

12. Schäfer GE, Rehbein C, Stiegler T, Hampl H. Renal anemia and its hemodynamic response-Findings invasively determined over a period of 20 years. Clin Nephrol 2002 Jul; 58 Suppl 1: S52-7.

13. G Bosi, R Crepaz, M R Gamberini, M Fortini, S Scarcia, E Bonsante et al. Left ventricular remodelling, and systolic and diastolic function in young adults with $\beta$ thalassaemia major: $\mathrm{A}$ Doppler echocardiographic assessment and correlation with haematological data. Heart 2003; 89: 762-766.

14. Valentin Fuster, R. Wayne Alexander, Robert A.O Rourke, Robert Roberts, Spencer B. King III, Ira S. Nash et al. Hurst's THE HEART. 11 th ed. New York: Mc Graw Hill companies; 2004.

15. G S Sainani, Philip Abraham, F. D. Dastur, V. R. Joshi, RD Lele, Late PJ Mehta, Sukumar Mukherjee, PS Shankar. A.P.I. Text book of Medicine. $6^{\text {th }}$ ed. Mumbai: Association of Physicians of India; 1999. 


\section{ORIGINAL ARTICLE}

\section{AUTHORS:}

1. Farquana Qushnood

2. Sanjay B. Bhagoji

3. Shireen Swaliha Quadri

4. Zaheera Sultana S.

5. Salim A. Dhundasi

6. K. K. Das

\section{PARTICULARS OF CONTRIBUTORS:}

1. Assistant Professor, Department of Physiology, Apollo Institute of Medical Sciences and Research, Hyderabad.

2. Assistant Professor, Department of Physiology, K.V.G Medical College \& Hospital, Kurunjibhag, Sullia.

3. Assistant Professor, Department of Physiology, SIMS, Shimoga.

4. Assistant Professor, Department of Physiology, Al Ameen Medical College, Bijapur.
5. Professor, Department of Physiology, Al Ameen Medical College, Bijapur.

6. Professor, Department of Physiology, Al Ameen Medical College, Bijapur.

\section{NAME ADDRESS EMAIL ID OF THE} CORRESPONDING AUTHOR:

Dr. Farquana Qushnood,

Assistant Professor,

Apollo Institute of Medical Sciences and Research, Jubilee Hills, Hyderabad.

Andhra Pradesh, India.

E-mail: dr.fqushnood@gmail.com

Date of Submission: 20/02/2014.

Date of Peer Review: 21/02/2014.

Date of Acceptance: 27/02/2014.

Date of Publishing: 12/03/2014. 